\title{
Increased Rates of Both Knee and Hip Arthroplasties in Older Patients with Ankylosing Spondylitis
}

\author{
Michael M. Ward (1)
}

ABSTRACT. Objective. To determine the risks of primary total hip arthroplasty (THA) and total knee arthroplasty (TKA) in older patients with ankylosing spondylitis (AS).

Methods. We used administrative data from 1999 to 2013 on US Medicare beneficiaries to identify patients ( $<75$ yrs old) with AS and a comparison group without AS. Rates of primary THA and primary TKA were computed for each group, and standardized for age, sex, and race. We also examined risks of primary TKA among patients with AS by their THA status.

Results. We analyzed 52,568 patients with AS and 4,617,179 patients without AS. Between 1999 and 2013, the standardized incidence of primary THA in patients with AS doubled from 4.5 per 1000 patient-years (PY) to 9.6 per 1000 PY. Rates of primary TKA were higher in patients with AS than controls in all years. In 2013, the standardized incidence of primary TKA in AS was 12.3 per 1000 PY versus 5.7 per $1000 \mathrm{PY}$ in the comparison group (RR 2.14, 95\% CI 1.93-2.38). Rates of primary TKA were twice as high among patients with AS and THA than among those without THA (20.4 vs 10.2 per $1000 \mathrm{PY})$.

Conclusion. Rates of THA in older patients with AS doubled over recent years, outpacing the increase in the general population. Rates of TKA were also substantially higher in older patients with AS. The increased risk of TKA in AS may be a consequence of damage from knee inflammation, or alterations in lower extremity biomechanics due to hip arthritis. (First Release July 1 2018; J Rheumatol 2019;46;31-7; doi:10.3899/jrheum.171316)

Key Indexing Terms: ANKYLOSING SPONDYLITIS
TOTAL KNEE ARTHROPLASTY

TOTAL HIP ARTHROPLASTY
Outside the axial skeleton, the hip is the joint most commonly affected by inflammatory arthritis in ankylosing spondylitis (AS). Up to $40 \%$ of patients in unselected cohorts report a history of symptomatic hip arthritis, and radiographic hip damage develops in up to $30 \%$ of patients ${ }^{1,2,3,4,5,6}$. In previous cohorts, $12 \%$ to $25 \%$ of patients progressed to total hip arthroplasty (THA) after 30 years of $\mathrm{AS}^{1}$. Most studies have been based at referral centers, which may include a higher proportion of more severely affected patients. An approach to better understand the magnitude of the problem of severe hip arthritis in AS would be to compare population-based rates of THA in patients with AS to rates of THA in the general population. Rates of THA in the general population in the United States have increased by $50 \%$ over the past decade, from 2 per 1000 patient-years (PY) in 1999 to 3 per $1000 \mathrm{PY}$ in $2007^{7,8}$. These increases may be attribu-

\footnotetext{
From the Intramural Research Program, US National Institute of Arthritis and Musculoskeletal and Skin Diseases (NIAMS), National Institutes of Health (NIH), Bethesda, Maryland, USA.

This study was funded by the Intramural Research Program, NIAMS, NIH (ZIA-AR-041153).

M.M. Ward, MD, MPH, Intramural Research Program, NIAMS, NIH. Address correspondence to Dr. M.M. Ward, NIAMS/NIH, Building 10 CRC, Room 4-1339, 10 Center Drive, Bethesda, Maryland 20892-1468, USA.E-mail:wardm1@mail.nih.gov

Accepted for publication March 29, 2018.
}

table in part to changing clinical indications and more liberal use of THA. It is not clear whether rates of THA in AS have increased in parallel with those in the general population, or if rates in AS have been stable over time, given that the clinical indications for THA in AS have not changed.

The knee is uncommonly affected by inflammatory arthritis in AS but may also be secondarily damaged if chronic hip arthritis alters lower extremity biomechanics 9,10 . Hip contractures that often develop after longstanding hip arthritis may induce knee contractures and altered gait ${ }^{11,12}$. Abnormal weight bearing, in the presence or absence of contractures and inflammation, may lead to knee damage necessitating arthroplasty ${ }^{13,14}$. Risks of total knee arthroplasty (TKA) in patients with AS, and the possible relationship between THA and TKA in AS, have not been investigated.

In our study, we used population-based data on US Medicare beneficiaries to compare rates of primary THA between older patients with AS and those without AS, and examined trends in these rates between 1999 and 2013. Additionally, we computed rates of primary TKA in older patients with AS and examined whether these rates differed between those with and without THA. We hypothesized that rates of primary THA would be higher in patients with AS than in the general population, would be stable over time, and that THA would be associated with higher risks of TKA.

Personal non-commercial use only. The Journal of Rheumatology Copyright @ 2019 . All rights reserved. 


\section{MATERIALS AND METHODS}

Data source and study design. Medicare is the federally funded universal medical insurance program for elderly Americans. Coverage typically begins at age 65 , although younger persons with certain permanent disabilities are also eligible. Coverage is retained until death, except for rare circumstances such as emigration or imprisonment. We used 100\% Medicare Part A and Part B fee-for-service data files from 1999 to 2013, which were the years of data currently available from the Centers for Medicare and Medicaid Services (CMS). Part A data includes information from all inpatient hospitalizations, including diagnosis codes (up to 25 possible fields per hospitalization) and procedure codes for surgeries and other interventions (up to 25 possible fields per hospitalization). Part B includes data on outpatient visits, including up to 13 possible diagnosis codes per visit. Diagnoses and procedures are coded in the data files using International Classification of Diseases, Ninth Revision, Clinical Modification (ICD-9-CM) codes. Data on intravenous medication use were available for all study years, and data on prescribed oral and injectable medications were available from Part D files starting in 2006. The study used a serial cross-sectional design, with rates of THA and TKA computed for each year.

The study protocol was approved by the US National Institute of Arthritis and Musculoskeletal and Skin Diseases (NIAMS) institutional review board (number 15-AR-N010). Data were made available by a data use agreement with CMS.

Identification of patients with AS. We searched all years for patients who had $\geq 2$ inpatient or outpatient diagnosis claims for AS (ICD-9 720.0) at least 7 days apart. Previous claims-based studies in the United States and United Kingdom reported that 2 claims with a code of 720.0 had a positive predictive value of 1.00 and 0.89 for AS, using a physician diagnosis as the reference standard ${ }^{15,16}$. To increase the specificity of our claims-based identification procedure, we counted only claims from face-to-face visits with a physician. In addition, we excluded patients who had $\geq 2$ diagnosis codes for rheumatoid arthritis (714.0) or psoriatic arthritis (696.0) at any time in their Medicare files.

Because AS typically begins in young adulthood, we considered patients to have prevalent AS from the time of entry into Medicare. We limited the study to those younger than 75 years, because the higher prevalence of osteoarthritis (OA) at older ages in both patients with and without AS may obscure any difference in THA risks related to $\mathrm{AS}^{15,17}$.

Because we were interested in rates of primary THA, we excluded patients whose first 2 recorded hip procedures in the data files were revision hip arthroplasties (ICD-9 00.70, 00.71, 00.72, 00.73, or 81.53). These patients likely had both primary THA before entering Medicare, but the dates of these procedures were unknown. We also excluded those whose THA was associated with hip fracture (ICD-9 820.X), because these THA would be unrelated to AS. Additionally, we included only those patients with full-year Part A and Part B coverage to ensure that all relevant diagnoses and procedures were recorded.

Comparison group. For comparison, we selected a $20 \%$ random sample of persons without AS in 1999 and supplemented this group with 5\% random samples of persons entering Medicare in subsequent years, so that patient entry into the comparison group was similar to the dynamic features of the AS cohort. We applied the same age, coverage, and procedure restrictions as in the AS group. We excluded patients with any diagnosis code for AS throughout their tenure in Medicare from this group. Matching was not used to select the comparison group.

Statistical analysis. Our outcomes of interest were primary THA (ICD-9 procedure code 81.51 ) and primary TKA (ICD-9 procedure code 81.54 ) for any reason (other than hip fracture). We focused on THA because partial hip arthroplasties made up only $2 \%$ of hip arthroplasties in patients with AS in this cohort. To calculate incidence rates, we first grouped patients with AS and those in the comparison group by calendar year, based on whether they were enrolled in Medicare and were age-eligible for inclusion in that year. For example, a person who entered Medicare at age 65 in 2002 would contribute to the denominator for the incidence rates in 2002 and thereafter, but not in earlier years. Similarly, a person aged 72 when the study started in 1999 would "age out" of the denominator after 2002, when they reached age 75 . Persons were also excluded from the study after their second primary THA.

For each year, we tabulated the number of primary THA (or TKA) in the AS and comparison groups, and calculated incidence rates based on the PY of observation in each group. Incidences were based on the surgery rather than the patient, so an individual patient could contribute $>1$ event to the incidence rates. Rates were age-, sex-, and race-standardized to the structure of the comparison group in 1999. Adjusted relative risks of THA (and TKA) in the AS group versus the comparison group were calculated for each year, which allowed examination of incidence trends over time. We repeated the analyses separately in men and women to determine whether sex modified these associations, given the higher prevalence of AS in men and higher rates of THA and TKA in women.

Next, we sorted patients with AS by THA status, and computed rates of TKA across study years in those with THA and those without THA, to determine whether the risks of these surgeries were associated. These rates were compared to rates of TKA in the comparison group without AS, with age-, sex-, and race-standardization to the structure of the comparison group across all study years.

SAS programs (version 9.4, SAS Institute) were used for data management and analysis. Significance was set at $\alpha=0.05,2$-tailed.

\section{RESULTS}

Patient groups. After applying the inclusion criteria, 52,568 patients with AS were analyzed (Figure 1). They had a mean of 9.3 visits coded as AS during time in Medicare. The mean \pm SD age of the AS group was $59.3 \pm 11.4$ years; $67.3 \%$ were men and $87.1 \%$ were white. The comparison group included $4,617,179$ persons, with a mean age of $65.7 \pm 10.0 ; 46.8 \%$ were men and $83.6 \%$ were white. The median number (range) of patients in the AS and comparison groups observed in any calendar year were $36,326(23,836-41,451)$ and $3,326,177$ $(2,866,856-4,617,179)$, respectively. There were 3243 primary THA in 2773 patients with AS, and 123,735 primary THA in 107,341 patients in the comparison group. Additionally, there were 4542 primary TKA in 3673 patients with AS, and 301,773 primary TKA in 250,296 patients in the comparison group. Sulfasalazine and methotrexate were each used by $<2 \%$ of patients with AS, and tumor necrosis factor- $\alpha$ (TNF- $\alpha$ ) inhibitor use was maximal in 2013 at 5.2\%.

Rates of primary THA. In 1999, standardized rates of primary THA in the AS and comparison groups were 4.6 and 1.9 per 1000 PY, respectively (Figure 2). The rate was substantially higher in patients with AS [relative risk (RR) $2.43,95 \%$ CI 2.00-2.96] in 1999. Rates of THA increased progressively in patients with AS through 2013, while rates increased in the comparison group through 2004, after which they stabilized. Adjusted RR increased over time, reaching 3.93 (95\% CI 3.49-4.43) in 2013.

Results were very similar in men and women (Figure 2). Rates of primary THA were somewhat higher among men with AS than women with AS, while rates of primary THA in the comparison group were lower in men than in women. Consequently, relative risks were somewhat higher among men than women in all years. In 2013, the adjusted relative risk was 4.47 in men and 3.71 in women (Figure 2).

Personal non-commercial use only. The Journal of Rheumatology Copyright @ 2019. All rights reserved 


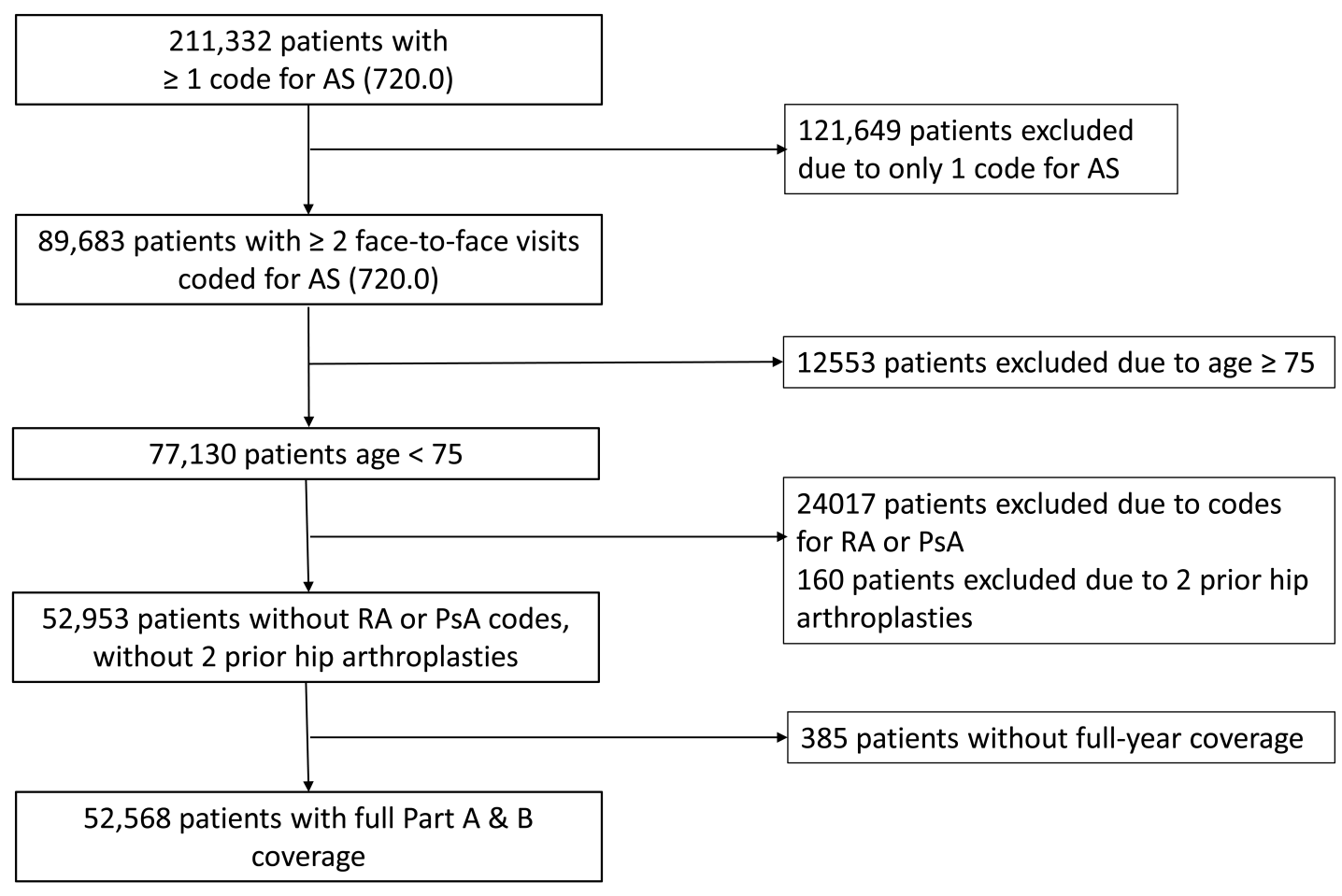

Figure 1. Identification of the ankylosing spondylitis study cohort. AS: ankylosing spondylitis; RA: rheumatoid arthritis; PsA: psoriatic arthritis.

Rates of primary TKA. Rates of primary TKA were higher than rates of primary THA in both patients with AS and patients in the comparison group (Figure 3). In 1999, standardized rates of primary TKA were 5.7 and 4.0 per 1000 $\mathrm{PY}$ in the AS and comparison groups, respectively. Rates increased in parallel in both groups through 2004, and then stabilized. Rates were persistently higher in AS, with adjusted RR increasing from 1.44 (95\% CI 1.21-1.71) in 1999 to 2.14 (95\% CI 1.93-2.38) in 2013.

Women had higher standardized rates of primary TKA than men, regardless of AS status (Figure 3). Between both men and women, rates were about twice as high among patients with AS than in the comparison group.

Risk of primary TKA by THA status. Among patients with AS and THA, the adjusted RR of primary TKA was 3.52 times higher than the risk of primary TKA in the comparison group (Table 1). The adjusted risk of primary TKA in AS patients without THA was also higher than in the comparison group, but RR was only 1.76 (Table 1 ). The adjusted risk of TKA was twice as high in patients with AS with THA than in patients with AS without THA. Results were similar in both men and women.

\section{DISCUSSION}

Our results confirm, in a population-based sample, that older patients with AS have higher rates of primary THA than persons without AS. This increased risk likely relates to the presence of inflammatory hip arthritis in AS, which may result in damage sufficient to require THA, or which may predispose to secondary hip OA. The data did not allow us to distinguish between these possible etiologies, and both likely contributed to the increased risk. Risks were elevated even in this older AS cohort. RR would be expected to be even higher in a younger cohort, given that THA is uncommon under age 65 in the general population ${ }^{17,18}$.

Over the study period, the incidence of primary THA in older patients with AS doubled and outpaced the increase in incidence in those without AS, so that RR increased progressively from 1999 to 2013. Reasons for this increase are unclear but may reflect wider use of THA among patients with AS after appreciation of acceptable frequencies of perioperative complications and good longterm outcomes ${ }^{19,20,21,22}$. These rates do not reflect any possible effects of antirheumatic medications, including TNF- $\alpha$ inhibitors, on rates of THA, because these medications were rarely used in this cohort. Low rates of treatment were speculated to account for the lack of decrease in arthroplasty rates in older patients with spondyloarthritis in a previous study ${ }^{23}$.

Our study documents for the first time, to our knowledge, an increased risk of primary TKA in older patients with AS. In 2009-2013, standardized rates of primary TKA were 2-fold higher in patients with AS than those without AS. Knee damage in AS may result from chronic inflammatory arthritis of the knee, or from mechanical stress because of altered weight bearing and lower extremity kinematics. Although inflammatory knee arthritis is a recognized feature of AS, it

Personal non-commercial use only. The Journal of Rheumatology Copyright @ 2019 . All rights reserved. 

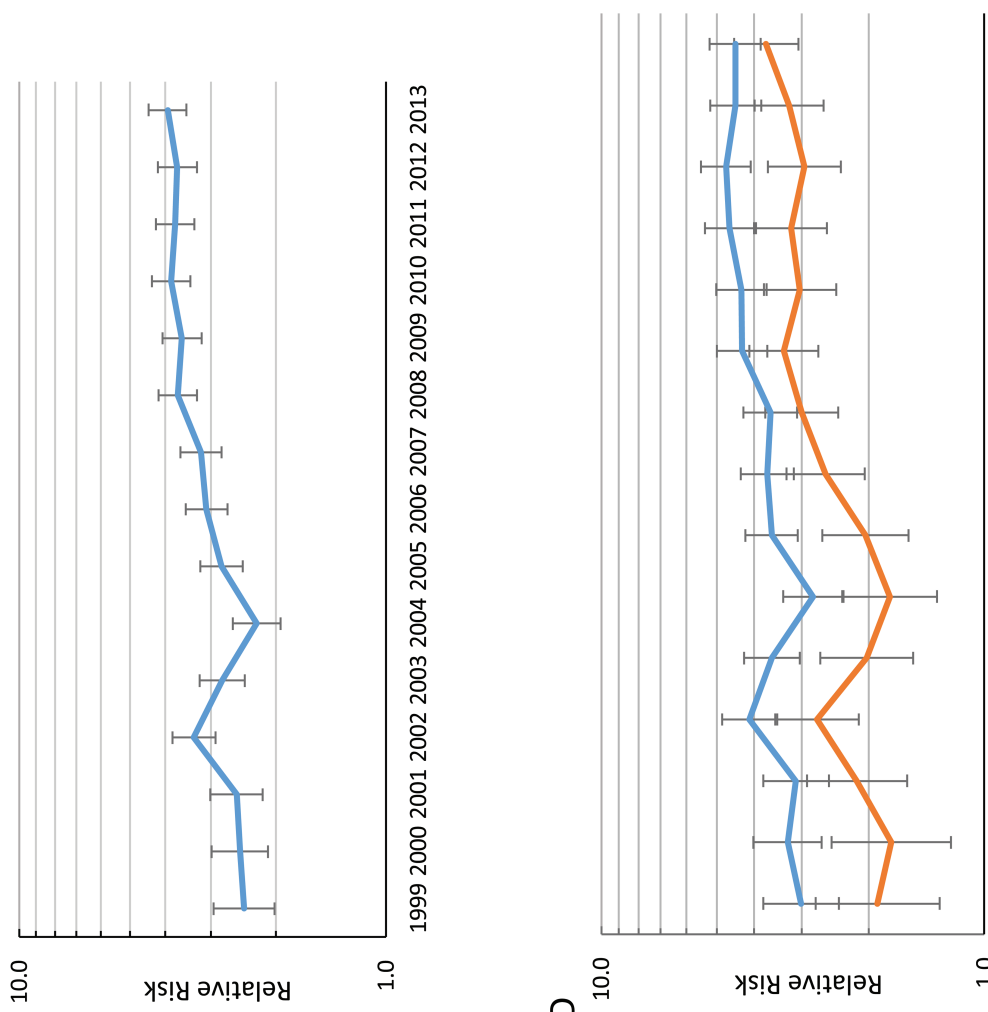

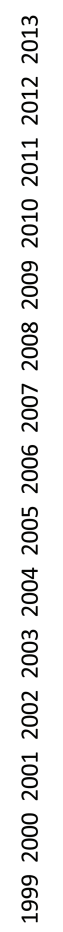

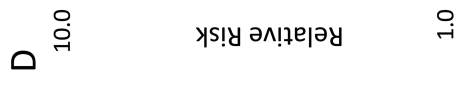

Figure 2. Incidence of total hip arthroplasty in older patients with AS and in the comparison group without AS, by year (A), and relative risks by year (B) overall, and among men and women $(\mathrm{C}$ and $\mathrm{D})$. Rates and relative risks were age-, sex-, and race-adjusted to the structure of the comparison group in 1999. Error bars represent $95 \%$ CI. In panel C, error bars for women with AS have circular end markers. Relative risk is plotted on a logarithmic scale. AS: ankylosing spondylitis; PY: patient-years. 


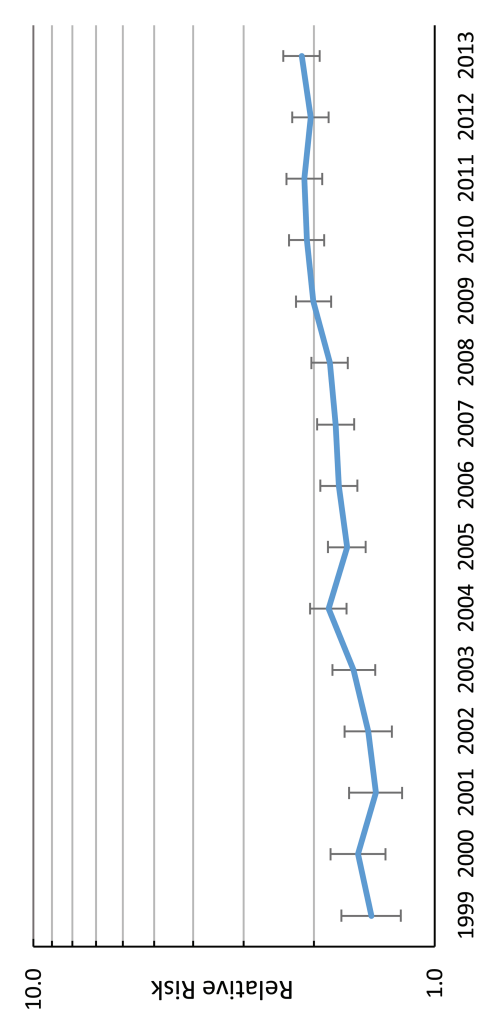

$\infty$

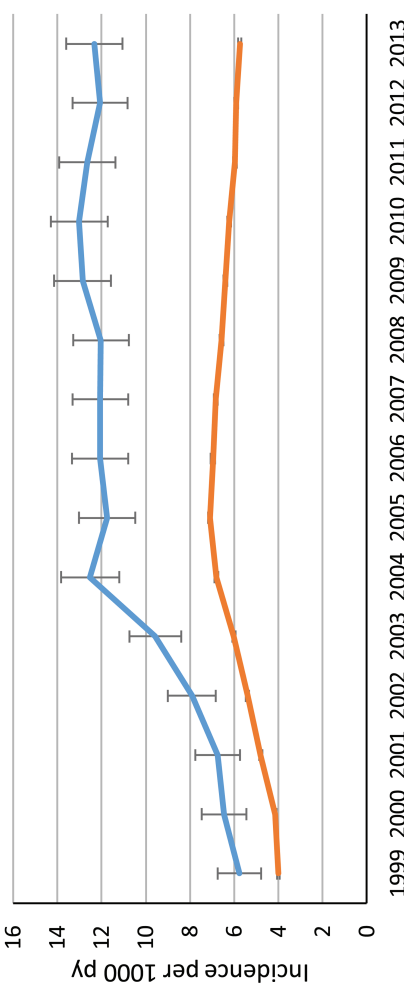

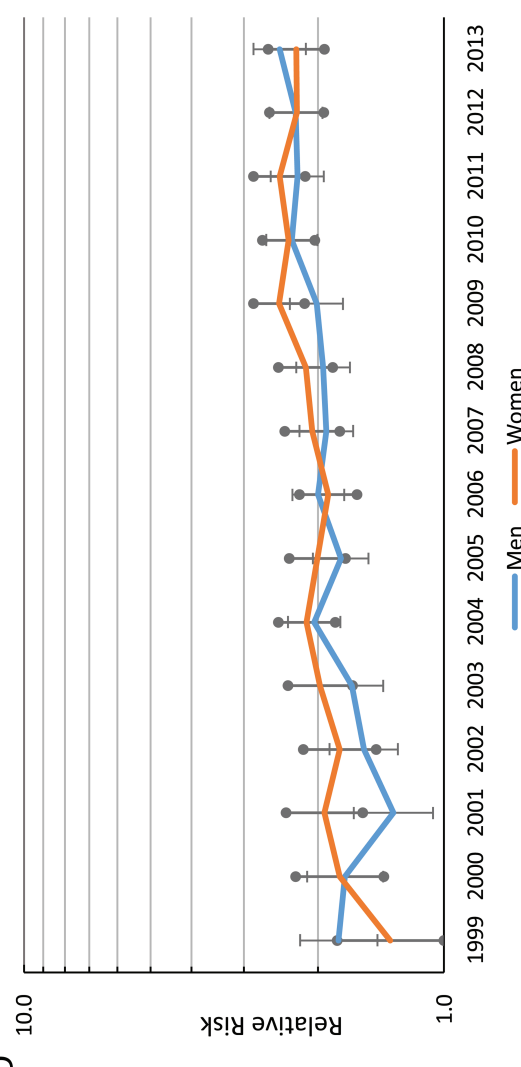

0
Figure 3. Incidence of total knee arthroplasty in older patients with $\mathrm{AS}$ and in the comparison group without AS, by year (A), and relative risks by year (B) overall, and among men and women (C and D). Rates and relative risks were age-, sex-, and race-adjusted to the structure of the comparison group in 1999. Error bars represent 95\% CI. In panel D, error bars for women have circular end markers. Relative risk is plotted on a logarithmic scale. AS: ankylosing spondylitis; PY: patient-years.

Personal non-commercial use only. The Journal of Rheumatology Copyright $\subset$ 2019. All rights reserved. 
Table 1. Incidence of total knee arthroplasties in older patients with AS by THA status.

\begin{tabular}{|c|c|c|}
\hline Patients & Incidence per $1000 \mathrm{PY}(95 \% \mathrm{CI})$ & RR $(95 \% \mathrm{CI})$ \\
\hline \multicolumn{3}{|l|}{ All } \\
\hline AS with THA & $20.4(18.7-22.3)$ & $3.52(3.23-3.84)$ \\
\hline AS without THA & $10.2(9.9-10.6)$ & $1.76(1.70-1.82)$ \\
\hline Comparison group & $5.8(5.7-5.9)$ & 1.00 \\
\hline \multicolumn{3}{|l|}{ Men } \\
\hline AS with THA & $16.8(14.8-18.8)$ & $4.01(3.58-4.50)$ \\
\hline AS without THA & $7.8(7.5-8.2)$ & $1.87(1.79-1.96)$ \\
\hline Comparison group & $4.2(4.1-4.3)$ & 1.00 \\
\hline \multicolumn{3}{|l|}{ Women } \\
\hline AS with THA & $28.3(24.6-31.9)$ & $3.88(3.41-4.42)$ \\
\hline AS without THA & $15.1(14.4-15.8)$ & $2.07(1.98-2.17)$ \\
\hline Comparison group & $7.3(7.2-7.3)$ & 1.00 \\
\hline
\end{tabular}

AS: ankylosing spondylitis; THA: total hip arthroplasty; PY: patient-years; RR: relative risk.

is not common ${ }^{9,24,25}$. Perhaps subclinical synovitis is present that over time leads to cartilage loss and secondary OA, or knee symptoms from subtle synovitis are misattributed to enthesitis. Dedicated studies are needed to address these possibilities.

The heightened risk of primary TKA among older patients with AS and THA could indicate that there is a subset of patients who have a predisposition to lower extremity synovitis that ultimately results in the need for multijoint replacements. Alternatively, the association between primary TKA and primary THA in patients with AS could reflect knee damage because of altered weight bearing to accommodate a painful, contracted, or ankylosed hip. The low prevalence of clinically evident knee synovitis in AS suggests that a mechanical cause is likely in most patients. However, these causes need not be mutually exclusive; both may be present and may synergize to cause knee damage. Older AS patients without THA also had an increased risk of TKA relative to those without AS, which may favor the knee inflammation hypothesis. However, we do not know whether these patients also had hip damage that was not yet severe enough to require arthroplasty.

Associations were comparable in both men and women, with the exception of somewhat lower RR for THA among women with AS relative to men. Finding comparable results in both sexes is important, given differences in rates of THA and TKA between women and men in the general population ${ }^{7,8}$. Additionally, given the diagnostic uncertainty inherent in any study of administrative data, we may be more confident in the results when they are replicated in both sexes, particularly if the diagnosis of AS was more accurate among men.

The strengths of our study include the large population-based sample, strict inclusion and exclusion based on diagnosis codes, and examination of trends in rates over 15 years. Arthroplasty rates in our comparison group matched those reported for Medicare beneficiaries in other studies, as did the secular trends in rates, supporting the validity of our analysis $^{8,26}$. However, our study also has several limitations. Because of the characteristics of Medicare eligibility, we could not examine rates of THA or TKA in younger patients. Although absolute rates may be lower at younger ages, RR in patients with AS compared to those without AS almost certainly would be higher than those reported here. Also, we do not know how many patients may have had arthroplasty prior to entry into Medicare. In one previous large series, the average age at THA among patients with AS was 56 years, so we may have recorded surgeries among most of the patients ${ }^{22}$. Our rates would be slight underestimates to the extent that patients with bilateral arthroplasties prior to enrollment in Medicare would contribute PY in our study but would not be eligible for a primary arthroplasty. The outcome was arthroplasty for any reason (other than hip fracture), because it was not possible to ascribe joint damage to inflammation, secondary OA, primary OA, or other indications based on these data. Data on laterality were not available, so we could not examine whether those who had both THA and TKA more likely had ipsilateral or contralateral procedures. Administrative data do not include clinical risk factors such as body mass index, so the influence of these factors cannot be examined. Last, as noted above, we relied on diagnosis codes for categorizing patients as having AS. Although these codes have been reported to have high positive predictive value for AS, some misclassification may have been present.

The finding of increased rates of TKA among older patients with AS indicates a need to better understand the possible causes of knee damage in AS, including possibly subclinical inflammation and mechanical factors. These results also raise questions about the appropriate timing of THA. THA is often postponed in younger patients with AS out of concern for the need for future revision hip arthroplasties. This strategy may need to be reconsidered if postponement of THA not only prolongs pain and disability, but also results in greater secondary knee damage and a need for TKA in the future.

Personal non-commercial use only. The Journal of Rheumatology Copyright $\subset$ $\subset$ 2019. All rights reserved 


\section{REFERENCES}

1. Vander Cruyssen B, Muñoz-Gomariz E, Font P, Mulero J, de Vlam $\mathrm{K}$, Boonen A, et al; ASPECT-REGISPONSER-RESPONDIA working group. Hip involvement in ankylosing spondylitis: epidemiology and risk factors associated with hip replacement surgery. Rheumatology 2010;49:73-81.

2. Sampaio-Barros PD, Bertolo MB, Kraemer MH, Neto JF, Samara AM. Primary ankylosing spondylitis: patterns of disease in a Brazilian population of 147 patients. J Rheumatol 2001;28:560-5.

3. Jeong H, Eun YH, Kim IY, Kim H, Lee J, Koh EM, et al. Characteristics of hip involvement in patients with ankylosing spondylitis in Korea. Korean J Intern Med 2017;32:158-64.

4. Brophy S, Mackay K, Al-Saidi A, Taylor G, Calin A. The natural history of ankylosing spondylitis defined by radiological progression. J Rheumatol 2002;29:1236-43.

5. Jang JH, Ward MM, Rucker AN, Reveille JD, Davis JC Jr, Weisman $\mathrm{MH}$, et al. Ankylosing spondylitis: patterns of radiographic involvement - a re-examination of accepted principles in a cohort of 769 patients. Radiology 2011;258:192-8.

6. Chen HA, Chen CH, Liao HT, Lin YJ, Chen PC, Chen WS, et al. Factors associated with radiographic spinal involvement and hip involvement in ankylosing spondylitis. Semin Arthritis Rheum 2011;40:552-8.

7. Cram P, Lu X, Kaboli PJ, Vaughan-Sarrazin MS, Cai X, Wolf BR, et al. Clinical characteristics and outcomes of Medicare patients undergoing total hip arthroplasty, 1991-2008. JAMA 2011;305:1560-7.

8. Singh JA, Lu X, Rosenthal GE, Ibrahim S, Cram P. Racial disparities in knee and hip total joint arthroplasty: an 18-year analysis of national Medicare data. Ann Rheum Dis 2014; 73:2107-15.

9. Parvizi J, Duffy GP, Trousdale RT. Total knee arthroplasty in patients with ankylosing spondylitis. J Bone Joint Surg Am 2001;83-A:1312-6.

10. Shakoor N, Hurwitz DE, Block JA, Shott S, Case JP. Asymmetric knee loading in advanced unilateral hip osteoarthritis. Arthritis Rheum 2003;48:1556-61.

11. McElwain JP, Sheehan JM. Bilateral hip and knee replacement for rheumatoid arthritis. J Bone Joint Surg Br 1985;67:261-5.

12. Goodman SM, Figgie M. Lower extremity arthroplasty in patients with inflammatory arthritis: preoperative and perioperative management. J Am Acad Orthop Surg 2013;21:355-63.

13. Shakoor N, Block JA, Shott S, Case JP. Nonrandom evolution of end-stage osteoarthritis of the lower limbs. Arthritis Rheum 2002;46:3185-9.

14. Shao Y, Zhang C, Charron KD, Macdonald SJ, McCalden RW, Bourne RB. The fate of the remaining knee(s) or hip(s) in osteoarthritic patients undergoing a primary TKA or THA.

J Arthroplasty 2013;28:1842-5.

15. Singh JA, Holmgren AR, Krug H, Noorbaloochi S. Accuracy of the diagnoses of spondylarthritides in Veterans Affairs medical center databases. Arthritis Rheum 2007;57:648-55.

16. Dubreuil M, Peloquin C, Zhang Y, Choi HK, Inman RD, Neogi T. Validity of ankylosing spondylitis diagnoses in The Health Improvement Network. Pharmacoepidemiol Drug Saf 2016; 25:399-404.

17. Kurtz S, Mowat F, Ong K, Chan N, Lau E, Halpern M. Prevalence of primary and revision total hip and knee arthroplasty in the United States from 1990 through 2002. J Bone Joint Surg Am 2005;87:1487-97.

18. Ibrahim T, Bloch B, Esler CN, Abrams KR, Harper WM. Temporal trends in primary total hip and knee arthroplasty surgery: results from a UK regional joint register, 1991-2004. Ann Roy Coll Surg Engl 2010;92:231-5.

19. Sochart DH, Porter ML. Long-term results of total hip replacement in young patients who had ankylosing spondylitis. Eighteen to thirty-year results with survivorship analysis. J Bone Joint Surg 1997;79:1181-9.

20. Sweeney S, Gupta R, Taylor G, Calin A. Total hip arthroplasty in ankylosing spondylitis: outcome in 340 patients. J Rheumatol 2001;28:1862-6.

21. Joshi AB, Markovic L, Hardinge K, Murphy JC. Total hip arthroplasty in ankylosing spondylitis: an analysis of 181 hips. J Arthroplasty 2002;17:427-33.

22. Schnaser EA, Browne JA, Padgett DE, Figgie MP, D’Apuzzo MR. Perioperative complications in patients with inflammatory arthropathy undergoing total hip arthroplasty. J Arthroplasty 2016;31:2286-90.

23. Mertelsmann-Voss C, Lyman S, Pan TJ, Goodman SM, Figgie MP, Mandl LA. US trends in rates of arthroplasty for inflammatory arthritis including rheumatoid arthritis, juvenile idiopathic arthritis, and spondyloarthritis. Arthritis Rheumatol 2014;66:1432-9.

24. Resnick D. Patterns of peripheral joint disease in ankylosing spondylitis. Radiology 1974;110:523-32.

25. Maksymowych WP, Jhangri GS, Lambert RG, Mallon C, Buenviaje $\mathrm{H}$, Pedrycz E, et al. Infliximab in ankylosing spondylitis: a prospective observational inception cohort analysis of efficacy and safety. J Rheumatol 2002;29:959-65.

26. Cram P, Lu X, Kates SL, Singh JA, Li Y, Wolf BR. Total knee arthroplasty volume, utilization, and outcomes among Medicare beneficiaries, 1991-2010. JAMA 2012;308:1227-36. 